\title{
Changes in Physical Activity and Sedentary Behaviors During COVID-19: Associations with Psychological Distress Among Mothers
}

\author{
Junia N de Brito $\mathbb{D}^{\prime}$ \\ Jessica K Friedman (D) \\ Rebecca L Emery ${ }^{2}$ \\ Dianne Neumark-Sztainer \\ Jerica M Berge (iD ${ }^{3}$ \\ Susan M Mason' \\ 'Division of Epidemiology and \\ Community Health, University of \\ Minnesota, Minneapolis, MN, USA; \\ ${ }^{2}$ Department of Family Medicine and \\ Biobehavioral Health, University of \\ Minnesota, Duluth, MN, USA; \\ ${ }^{3}$ Department of Family Medicine and \\ Community Health, University of \\ Minnesota, Minneapolis, MN, USA
}

Background: The coronavirus disease 2019 (COVID-19) pandemic increased mothers' caregiving demands, potentially placing them at increased risk for reduced engagement in healthful behaviors and high psychological distress.

Purpose: The purpose of this study was to describe perceived changes in moderate-vigorous physical activity (MVPA) and sedentary behaviors and the prevalence of different measures of psychological distress (depressive and anxiety symptoms, perceived stress, and positive and negative affect) among mothers. We also evaluated the associations of perceived change in MVPA and sedentary behaviors with measures of psychological distress.

Methods: We conducted a cross-sectional survey with 317 mothers living with children (020 years old) at home. Mothers self-reported perceived changes in MVPA and sedentary behaviors from before the pandemic to during COVID-19 stay-at-home orders and early reopening, and levels of depression and anxiety symptomatology, perceived stress, and positive and negative affect during the pandemic.

Results: Compared to pre-pandemic levels, $39 \%$ of mothers reported engaging less in MVPA, and $63 \%$ reported engaging in more sedentary activities. One-quarter and $31 \%$ of mothers reported moderate/severe depressive and anxiety symptoms, respectively, and 78\% medium/ high levels of perceived stress. Adjusted linear regression analyses revealed that reduced MVPA and increased sedentary behaviors were associated with high average scores of depressive and anxiety symptoms, perceived stress, negative affect, and low scores of positive affect.

Conclusion: A large proportion of mothers reported being less physically active and more engaged in sedentary behaviors, which were associated with increases in psychological distress. Clinical and public health intervention efforts should consider how to improve mothers' physical and mental health as the country emerges from this crisis.

Keywords: COVID-19, mothers, physical activity, stress, depression, anxiety

\section{Plain Language Summary}

1. What is the current understanding of this subject?

a. The COVID-19 pandemic resulted in unprecedented challenges for families. Because of gender roles and expectations of motherhood (eg, caretaking, housekeeping), mothers might be at increased risk for poor physical and mental health.

2. What does this study add to the literature?

a. This study fills a substantial knowledge gap on perceived changes in physical activity, sedentary behavior, and psychological distress among mothers with children across different developmental ages and stages of life, during COVID-19 stay-at-home orders and early reopening.
Division of Epidemiology and Community

Health, University of Minnesota, 1300

S 2nd St, Suite 300, Minneapolis, MN,

55455, USA

Tel +1 612-624-1818

Email nogue013@umn.edu 
3. What are the implications for public health practice?

a. Public health professionals should promote feasible strategies that encourage physical activity and provide mental health support to mothers during and after the COVID-19 pandemic.

\section{Introduction}

In early March 2020, the novel coronavirus disease 2019 (COVID-19), caused by the SARS-CoV-2 virus, was declared a pandemic by the World Health Organization. Days later, the United States declared a state of emergency for COVID-19. The associated public health safety measures implemented since the beginning of the pandemic to reduce the community spread of COVID-19 included stayat-home orders (ie, travel restrictions); physical distancing guidelines; and workplace, school, childcare, and recreational venue closures. Although necessary to control infection rates, these measures have resulted in unprecedented challenges to families with children, with many facing not only economic and financial disruptions but a dramatic shift in daily routines through the combination of workfrom-home requirements, reduced social support, and increased parenting and childcare demands. ${ }^{1}$ Because of the sociocultural dimensions and role expectations of gender and motherhood, and the fact that past outbreaks have further exacerbated long-standing gender inequalities and socioeconomic disparities, ${ }^{2}$ mothers' physical and mental health may be disproportionately and negatively affected during this crisis. ${ }^{3,4}$

Historically, women have been more likely than men to engage in lower levels of physical activity, ${ }^{5}$ higher levels of sedentary behaviors (eg, non-work sitting time), ${ }^{6}$ and suffer from poor mental health outcomes, including mood disorders (eg, depression), as well as high levels of anxiety, stress, and low mood. ${ }^{7-10}$ During the COVID-19 pandemic, further decreases in physical activity, increases in sedentary behavior, and increases in psychological distress have been particularly prominent among pregnant and new mothers. Research evidence from systematic reviews and meta-analyses showed that pregnant and postpartum women's engagement in physical activity (light, moderate and vigorous) have substantially decreased from before the COVID-19-related stay-at-home orders, and that they have experienced increased psychological distress during the pandemic. ${ }^{11,12}$ Closures of schools and daycare centers increased caregiving responsibilities at home, limiting available time and resources to engage in physical and emotional self-care routines. Aside from extensive anecdotal evidence circulated in mainstream media, there appears to be a substantial knowledge gap on changes in physical activity, sedentary behavior, and psychological distress among mothers with children across different developmental ages and stages of life.

The purpose of this study was to describe perceived changes in moderate-to-vigorous physical activity (MVPA) and sedentary behaviors from before the pandemic, and the prevalence of different measures of psychological distress (depressive symptoms, anxiety symptoms, perceived stress, and positive and negative affect) during the COVID-19 pandemic, among a population-based sample of mothers. Additionally, we aimed to cross-sectionally investigate whether these perceived changes in MVPA and sedentary behaviors were associated with these measures of psychological distress. Given the increased risk for unhealthy weight gain due to prolonged reductions in MVPA, increases in sedentary behaviors and mental distress, descriptive data about change in these health behaviors and psychological distress are needed to inform targeted public health and policy efforts to increase opportunities for physical, emotional, and mental self-care during and after the pandemic.

\section{Methods}

\section{Study Design and Sample}

Participants were members of the Life-course Experiences and Pregnancy (LEAP) study, a study of reproductive health among women participating in an existing longitudinal population-based study (Project EAT: Eating Among Teens and Young Adults). ${ }^{13}$ Data for the current analysis come from the COVID-19 Current Health study, which included women who (1) responded to the LEAP survey between August 2019 (when LEAP surveys were launched) and February 2020 (prior to the March national COVID-19 emergency declaration in the U.S.); (2) reported at least one live birth in the LEAP survey; (3) lived in a US state with a stay-at-home order due to the COVID-19 pandemic at some point between March and May 2020; and (4) had a valid email address. Of the 977 women who answered the LEAP survey, 461 women met these eligibility criteria. Invitations to participate in the COVID-19 Current Health survey were sent via email in late May 2020 and data were collected until mid-August 2020. A total of 317 women (69\% [317/461]) electronically consented and completed the online survey. The majority of participants were living in Minnesota (85\%) and, for most, stay-at-home orders were lifted or partially lifted $(94 \%)$ at the time they 
completed the COVID-19 Current Health survey. Participants received \$25 US incentives for completing the survey. The University of Minnesota's Institutional Review Board Human Subject's Committee approved all study protocols, and the study complies with the ethical standards of the Institution and with the 1964 Helsinki declaration and its later amendments or comparable ethical standards. Electronic informed consent was obtained from all participants.

\section{Measures}

The COVID-19 Current Health survey was designed to measure changes in health-related behaviors (eg, physical activity and sedentary behaviors and indicators of mothers' psychological distress [symptoms of depression and anxiety, perceived stress, and positive and negative affect]). Data about the number and age of children living in the home were collected, and sociodemographic data were available from the LEAP survey.

\section{Moderate to Vigorous Physical Activity}

Participants were asked, "During the past 30 days, how has the amount of moderate-intensity (hearts beating faster than normal and breathing harder than normal) or vigorous-intensity (heart beating much faster than normal, and breathing much harder than normal) physical activity changed?." Using a 5-item scale, participants could select one answer ("I have been engaged in MVPA:" 1: much less than usual, 2: less than usual, 3: same as usual, 4: more than usual, 5: much more than usual). To allow a sufficient number of participants in each category for statistical analyses, these answer options were categorized as follows: "much less than usual and less than usual," "same as usual," and "more than usual and much more than usual."

\section{Sedentary Behavior}

Participants were asked, "During the past 30 days, how has the amount you spend watching TV, browsing on your phone, or playing board games outside of work hours changed?." Answer options included a 5-item scale in which participants could select one answer option (I have been watching $\mathrm{TV}$, browsing on my phone, or playing board games outside of work hours: 1: much less than usual, 2: less than usual, 3: same as usual, 4: more than usual, 5: much more than usual). To allow a sufficient number of participants in each category, we categorized these answer options as follows: "much less than usual and less than usual," "same as usual," and "more than usual and much more than usual."

\section{Psychological Distress Depressive Symptoms}

Self-reported depressive symptoms were assessed using the 9-item Patient Health Questionnaire (PHQ-9). ${ }^{14}$ Participants were asked how often they have been bothered by nine common depressive symptoms (eg, "feeling down, depressed, or hopeless," "feeling tired or having little energy") during the previous two weeks. This instrument had four response options (1: not at all; 4: nearly every day). Scores were summed (range: 0-27), with higher scores indicating greater severity of depressive symptomatology.

\section{Anxiety Symptoms}

The 7-item self-reported Generalized Anxiety Disorder-7 (GAD-7) scale was used to measure anxiety symptoms. ${ }^{15}$ Participants were asked to rate how often they have been bothered by anxiety symptoms (eg, "feeling nervous, anxious, or on edge," "trouble relaxing") over the last two weeks using a 4-point Likert-scale (0: not at all; 3: nearly every day). Summed total scores ranged from 0 to 21 , with higher scores indicating higher severity levels of generalized anxiety disorder symptomology.

\section{Perceived Stress}

The 10-item Cohen's Perceived Stress Scale (PSS) evaluated perceived stress over the past month. ${ }^{16}$ This measure assessed participants' perceived stress levels on a 5-point Likert-scale (0: Never; 4: Very Often) as participants responded to items like "How often have you felt nervous and stressed?" and "How often have you been able to control irritations in your life?." Reverse scoring was employed for responses to positively stated items with the final score being the sum of all 10 responses. Scores on the PSS range from 0 to 40 , with higher scores denoting greater perceived stress.

\section{Positive and Negative Affect}

Affect assessments were completed using the 20-item Positive and Negative Affect Schedule (PANAS). ${ }^{17}$ Participants were asked to indicate to what extent they felt from the listed 10 positive (eg, "excited," "interested") and 10 negative affect (eg, "upset," "irritable") descriptions. Answers were reported using a 5-point Likert-scale (1: very slightly or not at all; 5: extremely). Scores for the positive and negative affect scales were summed (range 
10-50), with higher scores on both subscales representing higher positive or negative affect. Both the positive and negative affect subscales also have a midpoint score of 30 , which is equivalent to responding "moderately" to all subscale items.

\section{Statistical Analyses}

Linear regressions were used to examine the cross-sectional associations of perceived change in MVPA and sedentary behaviors (independent variables) with measures of psychological distress (dependent variables). Separate models were fit for each of the following measures of psychological distress: depressive symptoms, anxiety symptoms, perceived stress, positive affect, and negative affect. Based on an a priori list of potential confounding variables, models were adjusted for mother's race and ethnicity, annual household income, educational attainment, and employment status. The residuals for each model were plotted against predicted values and visually inspected to assess linear regression model assumptions. ${ }^{18}$ The inspection of the residuals indicated that the linear model assumptions were met. Data analyses were performed in Stata 16.1 (StataCorp LLC, College Station, TX).

\section{Results}

\section{Demographics}

Mothers included in our analytical sample ranged in age from 35 to 41 years old with an average age of $37.8(\mathrm{SD}=$ $1.4)$, were predominantly non-Hispanic White (74\%), well educated (Bachelor's degree or more: $58 \%$ ), married (75\%), employed fulltime before the pandemic (70\%), and had at least two children (ages 0-20 years) living at home during the pandemic (78\%). The full list of demographic characteristics of the analytic sample is presented in Table 1.

\section{Unadjusted Perceived Changes in Mothers' MVPA and Sedentary Behaviors and Mean Scores for Measures of Psychological Distress During COVID-19} Overall, 39\% of mothers reported engaging less in MVPA, $38 \%$ maintaining similar MVPA levels, and 23\% becoming more engaged in MVPA. For sedentary behaviors, $63 \%$ reported engaging in more sedentary activities, $27 \%$ maintaining a similar sedentary lifestyle, and $10 \%$ becoming less sedentary (Table 2). Approximately one-quarter of mothers in our sample reported moderate to severe depressive symptoms (PHQ-9 score $\geq 10$ ), one-third reported moderate to severe symptoms of anxiety (GAD-7 score $\geq 10$ ), and $78 \%$ reported medium to high levels of perceived stress (PSS score $\geq 14$ ) (cutoffs for PHQ-9, GAD7 , and PSS scores are not presented in a table). Mean scores for positive affect were slightly higher than negative affect and near the subscale midpoint of 30 (Table 2).

\section{Associations Between Perceived Changes in Mother's MVPA with Measures of Psychological Distress}

Relative to mothers who reported engaging in similar or more MVPA during the COVID-19 pandemic, mothers who reported engaging in less MVPA had higher average scores of depressive symptoms, anxiety symptoms, perceived stress, and negative affect, and lower average scores for positive affect, after controlling for sociodemographic factors (Table 3A).

\section{Associations Between Perceived Changes in Mother's Sedentary Behaviors with Measures of Psychological Distress}

After controlling for sociodemographic factors, mothers who reported engaging more in sedentary activities had higher average scores for depressive symptoms, anxiety symptoms, perceived stress, and negative affect, and lower scores for positive affect compared to mothers who reported similar (but not lower) levels of sedentary behaviors during the COVID-19 pandemic (Table 3B).

\section{Discussion}

Our study demonstrated that during the COVID-19 pandemic, the majority of mothers in our sample reported that their MVPA levels decreased or stayed the same while sedentary behaviors increased from before the pandemic. Additionally, a substantial number of mothers reported moderate to severe depressive and anxiety symptoms, most reported medium to high levels of perceived stress, and, on average, mothers reported slightly higher positive affect than negative affect. A perceived past-month decline in engagement in MVPA was associated with higher psychological distress relative to perceived past-month consistency in MVPA. Increased past-month engagement in sedentary behaviors was associated with higher psychological distress compared to mothers who reported similar, but not less engagement in sedentary activities. Support measures, such as increased access to health care and 
Table I Descriptive Sociodemographic Characteristics of a Sample of Mothers During the COVID-19 Pandemic, Mostly Residing in Minnesota, USA, May-August 2020 ( $N=317$ )

\begin{tabular}{|c|c|c|}
\hline Characteristics & $\mathbf{n}$ & $\%$ \\
\hline \multicolumn{3}{|l|}{ Race } \\
\hline African American & 17 & 5 \\
\hline American Indian/Alaska Native & 6 & 2 \\
\hline Asian & 39 & 13 \\
\hline Native Hawaiian/other Pacific Islander & I & I \\
\hline White & 233 & 74 \\
\hline Multi-racial & 7 & 2 \\
\hline \multicolumn{3}{|l|}{ Ethnicity } \\
\hline Hispanic/Latinx & 10 & 3 \\
\hline \multicolumn{3}{|l|}{ Educational attainment } \\
\hline Associate's degree or less & $13 \mid$ & 42 \\
\hline Bachelor's degree or more & 182 & 58 \\
\hline \multicolumn{3}{|l|}{ Employment status } \\
\hline Working full time & 222 & 70 \\
\hline Working part-time & 50 & 16 \\
\hline Out of the labor force & 44 & 14 \\
\hline \multicolumn{3}{|l|}{ Annual household income } \\
\hline$\$ 99,999$ or less & 153 & 49 \\
\hline$\$ 100,000$ or more & 160 & 51 \\
\hline \multicolumn{3}{|l|}{ Relationship status } \\
\hline Married, living with spouse & 237 & 75 \\
\hline Other* & 77 & 25 \\
\hline \multicolumn{3}{|l|}{ Number of children living at home } \\
\hline I & 68 & 22 \\
\hline 2 & 153 & 48 \\
\hline 3 or more & 96 & 30 \\
\hline \multicolumn{3}{|l|}{ Number of children by age of child } \\
\hline $0-5$ years & 95 & 30 \\
\hline $6-12$ years & 155 & 49 \\
\hline \multirow[t]{2}{*}{$13-20$ years } & 66 & 21 \\
\hline & Mean & SD \\
\hline Age, years (mean, sd) & 38.3 & 1.4 \\
\hline
\end{tabular}

Notes: For some characteristics, numbers may not add up to 317 because of missing responses or the "other" option was selected. *Includes other relationship arrangements (eg, unmarried, living with partner), separated, divorced, or single.

Abbreviations: SD, standard deviation; \%, percentage.

mental health services, aimed at preventing declines in healthy behaviors and mental health are important during and after the COVID-19 and future epidemics and pandemics to mitigate the long-term impacts in the health of mothers and their children.

The findings of high maternal psychological distress are consistent with one study that also documented a high
Table 2 Unadjusted Number of Respondents and Corresponding Percentages of Predictor Variables and Means, Standard Deviations, and Range of Outcome Variables Measured in a Sample of Mothers During the COVID-19 Pandemic, Mostly Residing in Minnesota, USA, MayAugust $2020(\mathrm{~N}=317)$

\begin{tabular}{|l|c|l|l|}
\hline Predictor Variables & $\mathbf{n}$ & $\%$ & \\
\hline Perceived change in MVPA & 124 & 39 & \\
Much less or less & 121 & 38 & \\
Same & 72 & 23 & \\
More or much more & & & \\
\hline Perceived change in sedentary behaviors & 33 & 10 & \\
Much less or less & 86 & 27 & \\
Same & 198 & 63 & \\
More or much more & Mean & SD & Range \\
\hline Dependent variables & 6.4 & 5.3 & $0-25$ \\
\hline PHQ-9 (Depressive symptoms) & 7.3 & 5.1 & $0-21$ \\
\hline GAD-7 (Anxiety symptoms) & 18.9 & 6.6 & $2-37$ \\
\hline PSS (Perceived stress) & & & \\
\hline PANAS (Affect) & 28.9 & 6.5 & $12-50$ \\
Positive affect & 24.4 & 7.6 & $10-49$ \\
\hline Negative affect &
\end{tabular}

Abbreviations: MVPA, moderate-vigorous physical activity; SD, standard deviation; $\%$, percentage.

prevalence of maternal depressive and anxiety symptoms among mothers of children aged 0-8 years during the COVID-19 pandemic. ${ }^{19}$ Moreover, our results are consistent with previous studies investigating the changes in physical activity, sedentary behaviors, and measures of psychological distress among pregnant and new mothers during COVID-19, ${ }^{11,12}$ thus, our study extends these findings into additional stages of motherhood (ie, among mothers with children across different developmental ages and stages of life).

The findings of our study also showed that mothers who reported maintaining or increasing their MVPA over the month prior to the survey, and those who maintained similar levels of sedentary behavior, reported lower psychological distress versus those who reported declining MVPA and increasing sedentary behaviors. These findings suggest that maintaining an active lifestyle is associated with lower psychological distress among mothers. The pandemic likely increased barriers for physical activity (eg, closure of gyms, indoor recreational spaces, and outdoor green spaces, increased caretaking responsibilities) and resulted in increased time spent in sedentary behaviors 
Table 3 Adjusted Associations Between Perceived Change in Moderate-Vigorous Physical Activity (MVPA) (A) and Sedentary Behaviors (B) and Psychological Distress Measures in a Sample of Mothers During the COVID-19 Pandemic, Mostly Residing in Minnesota, USA, May-August 2020 ( $\mathrm{N}=317$ )

\begin{tabular}{|c|c|c|c|c|c|c|}
\hline \multicolumn{7}{|l|}{ A. MVPA } \\
\hline \multirow{3}{*}{$\begin{array}{l}\text { Psychological outcome } \\
\text { measures }\end{array}$} & \multicolumn{6}{|c|}{ MVPA } \\
\hline & \multicolumn{2}{|c|}{ Much less or less $(n=\mid 24)$} & \multicolumn{2}{|c|}{ Same $(n=|2|)$} & \multicolumn{2}{|c|}{ More or much more $(n=72)$} \\
\hline & $\begin{array}{l}\text { Predicted } \\
\text { mean }\end{array}$ & $95 \% \mathrm{Cl}$ & $\begin{array}{l}\text { Predicted } \\
\text { mean }\end{array}$ & $95 \% \mathrm{Cl}$ & $\begin{array}{l}\text { Predicted } \\
\text { mean }\end{array}$ & $95 \% \mathrm{Cl}$ \\
\hline Depressive symptoms & 7.7 & $6.7,8.6^{\mathrm{a}}$ & 5.6 & $4.6,6.5^{b}$ & 5.8 & $4.5,7.0^{\mathrm{b}}$ \\
\hline Anxiety symptoms & 8.2 & $7.3,9.1^{a}$ & 6.9 & $6.0,7.8^{\mathrm{b}}$ & 6.6 & $5.4,7.7^{\mathrm{b}}$ \\
\hline Perceived stress & 20.5 & $19.3,21.7^{\mathrm{a}}$ & 17.9 & $16.7,19.1^{\mathrm{b}}$ & 17.9 & $16.4,19.5^{\mathrm{b}}$ \\
\hline Positive affect & 26.6 & $25.5,27.8^{\mathrm{a}}$ & 30.6 & $29.5,31.7^{b}$ & 29.8 & $28.3,31.3^{b}$ \\
\hline Negative affect & 25.9 & $24.5,27.2^{\mathrm{a}}$ & 23.4 & $22.0,24.7^{b}$ & 23.5 & $21.7,25.2^{b}$ \\
\hline \multicolumn{7}{|l|}{ B. Sedentary behaviors } \\
\hline & \multicolumn{6}{|c|}{ Sedentary behaviors } \\
\hline \multirow{2}{*}{$\begin{array}{l}\text { Psychological outcome } \\
\text { measures }\end{array}$} & \multicolumn{2}{|c|}{ Much less or less $(n=33)$} & \multicolumn{2}{|c|}{ Same $(n=86)$} & \multicolumn{2}{|c|}{ More or much more $(n=198)$} \\
\hline & $\begin{array}{l}\text { Predicted } \\
\text { mean }\end{array}$ & $95 \% \mathrm{Cl}$ & $\begin{array}{l}\text { Predicted } \\
\text { mean }\end{array}$ & $95 \% \mathrm{Cl}$ & $\begin{array}{l}\text { Predicted } \\
\text { mean }\end{array}$ & $95 \% \mathrm{Cl}$ \\
\hline Depressive symptoms & 7.9 & $6.1,9.7^{\mathrm{a}}$ & 4.7 & $3.6,5.8^{\mathrm{b}}$ & 6.9 & $6.2,7.6^{\mathrm{a}}$ \\
\hline Anxiety symptoms & 7.7 & $5.9,9.4^{\mathrm{a}, \mathrm{b}}$ & 6.3 & $5.2,7.4^{\mathrm{a}}$ & 7.7 & $7.0,8.4^{b}$ \\
\hline Perceived stress & 18.5 & $16.1,20.8^{a, b}$ & 16.8 & $15.4,18.2^{\mathrm{a}}$ & 19.9 & $19.0,20.9^{b}$ \\
\hline Positive affect & 30.1 & $27.9,32.4^{\mathrm{a}}$ & 31.2 & $29.8,32.5^{\mathrm{a}}$ & 27.6 & $26.7,28.5^{b}$ \\
\hline Negative affect & 23.7 & $21.1,26.3^{a, b}$ & 22.4 & $20.8,24.0^{\mathrm{a}}$ & 25.3 & $24.3,26.4^{b}$ \\
\hline
\end{tabular}

Notes: Final sample size for each model: $n=293$, depressive symptoms model; $n=296$, anxiety symptoms model; $n=299$, perceived stress model; $n=300$, positive and negative affect models. Predicted means in a row without a common superscript letter statistically differ $(P<0.05)$.

Abbreviations: MVPA, moderate-vigorous physical activity; $95 \% \mathrm{Cl}, 95 \%$ confidence interval.

(eg, increased work and leisure screen time). Clinicians and public health professionals play a critical role in connecting mothers to mental help support and providing affordable and feasible alternative activities to increase physical activity and reduce sedentary behaviors for improved mental health. These activities might include engaging in active family playtime, taking online fitness classes via free health applications, doing home improvement projects, gardening, and going for walks. Additionally, the lack of maternal role modeling physical activity and maternal mental health disorders may have a lasting adverse influence on children. Thus, the implementation of tailored interventions that promote engagement in physical activity, reduction of sedentary behaviors, and reduction and management of psychological distress can help prevent poor long-term engagement in health behaviors and psychopathologies among both mothers and children.

These results should be interpreted in light of several limitations. First, the cross-sectional study design hindered our ability to establish temporality and therefore there is a possibility of reverse causation (influence of psychological distress on engagement in MVPA and sedentary behaviors). Although our study incorporated perceived change from before to during the pandemic to capture the retrospective evolution of MVPA and sedentary behaviors, and surveyed a non-clinical sample of mothers, prior studies have shown a bidirectional relationship between physical activity, sedentary behavior, and psychological distress. ${ }^{20}$ Other studies are encouraged to investigate these bidirectional associations, particularly among mothers with clinical psychological distress as they may be more likely to benefit from physical activity, sedentary behavior, mental health support interventions. Second, all measures were self-reported and thus prone to measurement errors. Moreover, the assessment of MVPA and sedentary behaviors reflect their perceived change in the past 30 days, whereas the measures of psychological distress reflect their assessment in the period of the previous two weeks. It is possible that perceived change in these 
behaviors was not synchronized with the measures of psychological distress, which could undermine our ability to observe a true association. Third, the generalizability of our findings is limited to non-Hispanic White mothers mostly residing in Minnesota, US. Other research should examine whether our findings are replicated in a more socioeconomically, racially, ethnically, and geographically diverse sample of mothers who are likely at the greatest risk for further reduced engagement in physical activity and other health behaviors, and higher psychological distress.

\section{Conclusions}

During the COVID-19 pandemic, a large proportion of mothers reported being less physically active and more engaged in sedentary behaviors, which were associated with increased levels of psychological distress. Given the physical and mental health benefits of physical activity, strategies to that reduce barriers and encourage physical activity and provide mental health support amidst the demands of parenting should be promoted. This approach should consider individual, organizational, community, and policy-level interventions that support both physical activity and mental health. These strategies are critical now more than ever as we emerge from the personal and societal-level impacts of the COVID-19 pandemic.

\section{Acknowledgments}

The authors thank the LEAP staff and study participants for their important contributions.

\section{Funding}

Data collection for the study was supported by Grant Number R01HL116892 from the National Heart, Lung, and Blood Institute (NHLBI) (PI: D Neumark-Sztainer), R01HD090053 from the Eunice Kennedy Shriver National Institute of Child Health and Human Development (NICHD) (PI: SM Mason), and 20SGPSR-1YR10FdB from University of Minnesota's Healthy Foods, Healthy Lives Institute (Co-PIs: JN de Brito, JK Friedman). Training for the first author ( $\mathrm{JN}$ de Brito) was supported by the NHLBI-Ruth L. Kirschstein National Research Service Award T32 Research on Eating and Activity for Community Health (REACH) Applied Epidemiology Training Program (T32HL150452; PI: D NeumarkSztainer). Jessica K Friedman's time was supported by the NICHD under R01HD090053 (PI: SM Mason). Rebecca Emery's time was supported by the National
Center for Advancing Translational Sciences under TL1 TR002493 (PI: Fulkerson) and UL1 TR002494 (PI: Blazar).

\section{Disclosure}

The authors have no conflicts of interest to declare.

\section{References}

1. Venta A, Bick J, Bechelli J. COVID-19 threatens maternal mental health and infant development: possible paths from stress and isolation to adverse outcomes and a call for research and practice. Child Psychiatry Hum Dev. 2021;52(2):200-204. doi:10.1007/s10578-021-01140-7

2. Wenham C, Smith J, Morgan R. COVID-19: the gendered impacts of the outbreak. Lancet. 2020;395(10227):846-848. doi:10.1016/S01406736(20)30526-2

3. Roy SS, Foraker RE, Girton RA, Mansfield AJ, Posttraumatic stress disorder and incident heart failure among a community-based sample of US veterans. Am J Public Heal. 2015;105(4):757-763. doi:10.2105/AJPH.2014.302342

4. Tison GH, Avram R, Kuhar P. Worldwide effect of COVID-19 on physical activity: a descriptive study. Ann Intern Med. 2020;173 (9):767-770. doi:10.7326/M20-2665

5. Troiano RP, Berrigan D, Dodd KW, Mâsse LC, Tilert T, Mcdowell M. Physical activity in the United States measured by accelerometer. Med Sci Sports Exerc. 2008;40(1):181-188. doi:10.1249/mss.0b013e31815a51b3

6. Van Uffelen JGZ, Van Gellecum YR, Burton NW, Peeters G, Heesch KC, Brown WJ. Sitting-time, physical activity, and depressive symptoms in mid-aged women. Am J Prev Med. 2013;45 (3):276-281. doi:10.1016/j.amepre.2013.04.009

7. Bangasser DA. Sex differences in stress-related receptors: "Micro" differences with "macro" implications for mood and anxiety disorders. Biol Sex Differ. 2013;4(1):1-13. doi:10.1186/2042-6410-4-2

8. Hyde JS, Mezulis AH, Abramson LY. The ABCs of depression: integrating affective, biological, and cognitive models to explain the emergence of the gender difference in depression. Psychol Rev. 2008;115(2):291-313. doi:10.1037/0033-295X.115.2.291

9. Balhara YS, Verma R, Gupta C. Gender differences in stress response: role of developmental and biological determinants. Ind Psychiatry J. 2012;20(1):4. doi:10.4103/0972-6748.98407

10. Brody DJ, Pratt LA, Hughes JP. Prevalence of depression among adults aged 20 and over: United States, 2013-2016. NCHS Data Brief, No 303; 2018. Available from: https://www.cdc.gov/nchs/ data/databriefs/db303 table.pdf\#3. Accessed May 18, 2021.

11. Yan H, Ding Y, Guo W. Mental health of pregnant and postpartum women during the coronavirus disease 2019 pandemic: a systematic review and meta-analysis. Front Psychol. 2020;11. doi:10.3389/fpsyg.2020.617001

12. Hessami K, Romanelli C, Chiurazzi M, Cozzolino M. COVID-19 pandemic and maternal mental health: a systematic review and meta-analysis. J Matern Neonatal Med. 2020:1-8. doi:10.1080/ 14767058.2020.1843155

13. Watts AW, Mason SM, Loth K, Larson N, Neumark-Sztainer D. Socioeconomic differences in overweight and weight-related behaviors across adolescence and young adulthood: 10-year longitudinal findings from project EAT. Prev Med. 2016;87:194-199. doi:10.1016/ j.ypmed.2016.03.007

14. Naik AD, Martin LA, Karel M, et al. Cancer survivor rehabilitation and recovery: protocol for the Veterans Cancer Rehabilitation Study (Vet-CaRes). BMC Heal Serv Res. 2013;13:93. doi:10.1186/14726963-13-93

15. Spitzer RL, Kroenke K, Williams JBW, Löwe B. A brief measure for assessing generalized anxiety disorder: the GAD-7. Arch Intern Med. 2006;166(10):1092-1097. doi:10.1001/archinte.166.10.1092 
16. Cohen S, Kamarck T, Mermelstein R. A global measure of perceived stress. J Health Soc Behav. 1983;24(4):385-396. doi:10.1016/j. socscimed.2016.08.021

17. Watson D, Clark LA, Tellegen A. Development and validation of brief measures of positive and negative affect: the PANAS scales. $J$ Pers Soc Psychol. 1988;54(6):1063-1070. doi:10.1037/00223514.54.6.1063

18. Casson RJ, Farmer LDM. Understanding and checking the assumptions of linear regression: a primer for medical researchers. Clin Exp Ophthalmol. 2014;42(6):590-596. doi:10.11 $11 /$ ceo. 12358
19. Cameron EE, Joyce KM, Delaquis CP, Reynolds K, Protudjer JLP, Roos LE. Maternal psychological distress \& mental health service use during the COVID-19 pandemic. J Affect Disord. 2020;276 (July):765-774. doi:10.1016/j.jad.2020.07.081

20. Da Silva MA, Singh-Manoux A, Brunner EJ, et al. Bidirectional association between physical activity and symptoms of anxiety and depression: the Whitehall II study. Eur J Epidemiol. 2012;27 (7):537-546. doi:10.1007/s10654-012-9692-8

\section{Publish your work in this journal}

The International Journal of Women's Health is an international, peerreviewed open-access journal publishing original research, reports, editorials, reviews and commentaries on all aspects of women's healthcare including gynecology, obstetrics, and breast cancer. The manuscript management system is completely online and includes a very quick and fair peer-review system, which is all easy to use. Visit http://www.dovepress.com/testimonials.php to read real quotes from published authors. 